\title{
REACCIÓN DE GERMOPLASMA DE SOYA A Phakopsora pachyrhizi EN CAMPO
}

\author{
REACTION OF SOYBEAN GERMPLASM TO Phakopsora pachyrhizi IN THE FIELD
}

\author{
María de los Ángeles Peña-del-Río ${ }^{1 \star}$, Nicolás Maldonado-Moreno² y Arturo Díaz-Franco ${ }^{3}$
}

\begin{abstract}
${ }^{1}$ Campo Experimental General Terán, Instituto Nacional de Investigaciones Forestales, Agrícolas y Pecuarias (INIFAP). Km 31 Carr. Montemorelos-China. 67400, General Terán, Nuevo León, México. ${ }^{2}$ Campo Experimental Las Huastecas, INIFAP. Km 55 Carr. Tampico-Mante. 89610, Altamira, Tamaulipas, México. ${ }^{3}$ Campo Experimental Río Bravo, INIFAP. Km 61 Carr. Matamoros-Reynosa. 88900, Río Bravo, Tamaulipas, México.

${ }^{*}$ Autor para correspondencia (angelesrio36@yahoo.com.mx).
\end{abstract}

\section{RESUMEN}

La roya asiática (Phakopsora pachyrhizi) de la soya (Glycine max (L.) Merr.), hizo su aparición en 2005 en México. Las variedades comerciales comúnmente sembradas han mostrado susceptibilidad al patógeno, por lo que el presente estudio tuvo el objetivo buscar fuentes de resistencia genética a la enfermedad, a través de la reacción de 197 genotipos de soya evaluados en campo. Se establecieron dos fechas de siembra (24 de febrero y 24 de abril, 2007) y las inoculaciones se hicieron a inicio de la floración. La severidad se evaluó en la etapa R6, mediante una escala de $1(0 \%)$ a $3(>30 \%)$. Se identificaron 23 genotipos de soya resistentes a $P$. pachyrhizi, en ambas fechas de siembra, los cuales no exhibieron lesiones foliares.

Palabras clave: Glycine max, roya asiática, genotipos resistentes.

\section{SUMMARY}

Asian soybean rust (Phakopsora pachyrhizi), appeared in México in 2005. Commonly sowed commercial cultivars have shown susceptibility to the pathogen, therefore this study searched for sources of genetic resistance to the disease by field evaluation of 197 soybean genotypes. Plants were sowed at two planting dates (24-Feb and 24-Apr, $2007)$ and inoculated during flowering initiation. Disease severity was measured during the $\mathrm{R} 6$ stage, using a rating scale from $1(0 \%)$ to 3 (> $30 \%)$. Twenty-three soybean genotypes were identified as resistant to $P$. pachyrhizi and did not show leaf damage at either planting date..

Index words: Glycine max, Asian soybean rust, resistant genotypes.

\section{INTRODUCCIÓN}

Phakopsora pachyrhizi (H. Sydow \& Sydow) es el agente causal de la roya asiática de la soya [Glycine max (L.) Merr.] y es económicamente importante para el cultivo en condiciones ambientales favorables. Por mucho tiempo el patógeno estuvo confinado en países asiáticos; después, y cronológicamente, hizo su aparición en África entre 1996-2001, en América del sur en 2002, en 2004 en Estados Unidos, y en México en 2005 (Schneider et al., 2005; Yorinori et al., 2005 Cárcamo-Rodríguez y Aguilar-Ríos, 2006).

La soya en México, con una producción de 247,500 t en el 2012 (SIAP, 2014), sólo cubre 7 \% de la demanda nacional.
La principal región productora es la Planicie Huasteca, que comprende el sur de Tamaulipas, oriente de San Luis Potosí y norte de Veracruz. La roya asiática de la soya se detectó por primera vez en la Planicie Huasteca en 2005, donde la producción se redujo más de $80 \%$ en los lotes donde ocurrió la enfermedad (Cárcamo-Rodríguez y Aguilar-Ríos, 2006; Terán-Vargas et al., 2007); de aquí, P. pachyrhizi se ha diseminado a otras regiones del país. Las principales variedades de soya sembradas en México son 'Huasteca 100', 'Huasteca 200', 'Huasteca 300' y 'Huasteca 400', las cuales han mostrado susceptibilidad al patógeno (Terán-Vargas et al., 2007).

Lograr la resistencia genética a $P$. pachyrhizi es la mejor estrategia a largo plazo para la prevención de la roya asiática; existe limitada información sobre su resistencia (Pham et al., 2009) y en México no hay antecedentes. Las condiciones óptimas para el desarrollo de la roya asiática son temperaturas entre 18 a $25{ }^{\circ} \mathrm{C}$ y humedad relativa superior a 75 \% (Terán-Vargas et al., 2007; Koenning et al., 2008). Se ha demostrado que la enfermedad puede presentarse en la soya en cualquier etapa de su desarrollo (Twizeyimana et al., 2007; Miles et al., 2008), sin embargo, lo más común es la presencia en las hojas inferiores a partir del inicio de la etapa reproductiva (R1), y su intensidad se incrementa a medida que avanza la edad de la planta (Kawuki et al., 2004; Terán-Vargas et al., 2007; Koenning et al., 2008).

$\mathrm{Al}$ principio se observan pequeñas lesiones amarillentas en el haz de la hoja, peciolos y tallos, al avanzar la enfermedad hacia el tercio medio y superior de la planta, las hojas se tornan amarillentas y las lesiones multiplicadas se manifiestan como pústulas de color marrón-amarillento a marrón-rojizo, cuando también ocurre una defoliación prematura de la planta (Terán-Vargas et al., 2007; Koenning et al., 2008; Giesler, 2009). El presente estudio tuvo como objetivo buscar fuentes de resistencia genética a la roya asiática, a través de la reacción de 197 genotipos de soya evaluados en campo. 


\section{MATERIALES Y MÉTODOS}

Localidad y germoplasma. El estudio se realizó en terrenos de General Terán en el Estado de Nuevo León (25 $18^{\prime} 5^{\prime \prime} \mathrm{LN}, 99^{\circ} 35^{\prime} 35^{\prime} \mathrm{LO}$ ), región semicálida y subhúmeda. El germoplasma de soya utilizado fueron 197 genotipos; 61 líneas avanzadas sobresalientes del Programa de Mejoramiento Genético de Soya del Campo Experimental Las Huastecas, del Instituto Nacional de Investigaciones Forestales, Agrícolas y Pecuarias (INIFAP), y 132 genotipos provenientes del Departamento de Agricultura de los Estados Unidos. Se utilizaron como testigos susceptibles a las variedades comerciales 'Huasteca 100', 'Huasteca 200', 'Huasteca 300' y 'Huasteca 400' (Maldonado-Moreno, 1994; Maldonado-Moreno y Ascencio-Luciano, 2005a; Maldonado-Moreno y Ascencio-Luciano, 2005b).

Preparación del inóculo. El inóculo de P. pachyrhizi se obtuvo en noviembre de 2006 de una mezcla de urediosporas colectadas de pústulas en hojas de soya severamente infectadas, procedentes de Tamaulipas y Nuevo León (Figura 1), las cuales se depositaron en cajas Petri y se almacenaron en bolsas de papel a $5{ }^{\circ} \mathrm{C}$. Para la inoculación en el campo, se utilizó una suspensión de urediosporas en agua destilada con $0.1 \%$ de Tween 20 (monolaurato de sodio), a una concentración de $2.5 \times 10^{3}$ esporas $\mathrm{mL}^{-1}$, calculada en cámara de Nuebauer ${ }^{\circledR}$.

Manejo de campo. Los genotipos de soya se sembraron en forma manual en parcelas de un surco de $1 \mathrm{~m}$ de longitud y $0.8 \mathrm{~m}$ de separación, con 25 semillas por surco; cada 20 genotipos se intercalaron dos surcos con las variedades comerciales susceptibles para promover una dispersión del inóculo en las parcelas. Los ensayos se establecieron en dos fechas, el 24 de febrero y 24 de abril del 2007. Las inoculaciones en campo se realizaron asperjando las plantas con la suspensión de urediosporas al inicio de la floración, 70 y 50 dds del 24 de febrero y del 24 de abril, respectivamente; en ambos casos a las 5:00 am y en condiciones de rocío. Este factor es determinante para la germinación de las esporas (Bonde et al., 2007).

Para mantener un microclima favorable durante la etapa reproductiva $(R)$ y según las condiciones de humedad en el suelo, se aplicaron riegos aproximadamente cada $10 \mathrm{~d}$. La severidad de la roya asiática en el germoplasma de soya se evaluó en función del nivel de la densidad (\%) de las lesiones en las hojas medida con una escala de 1 (resistente $0 \%$ ) a 3 (susceptible $>30 \%$ ) (Miles et al., 2008), y en la etapa de desarrollo reproductivo R6, tal como lo indicaron Kawuki et al. (2004), Twizeyimana et al. (2007) y Oloka et al. (2008).

\section{RESULTADOS Y DISCUSIÓN}

En ambas fechas de siembra, las primeras lesiones en las hojas se observaron a los $6 \mathrm{~d}$ después de la inoculación. Resultó evidente que el desarrollo de la enfermedad se incrementó de acuerdo con la precocidad de las líneas, ya que la severidad aumentó conforme avanzó a la etapa reproductiva de la planta. Este fenómeno también fue observado por Kawuki et al. (2004) y Koenning et al. (2008), quienes señalaron que la severidad de la roya en la soya se incrementaba con la edad del cultivo.

La línea 'H02-1531', por su precocidad y susceptibilidad, fue la primera en manifestar lesiones con abundante esporulación. Las cuatro variedades testigo también mostraron una severa infección homogénea (entre 80 y 100 \%) en todos los surcos que fueron intercalados. Esto indicó que hubo una uniforme dispersión y presión del patógeno en el campo.

En total se identificaron 23 genotipos de soya resistentes a $P$. pachyrhizi, en ambas fechas de siembra, las cuales no exhibieron lesiones foliares. Estas fueron: 'L85-2378', 'PI 230970', 'AkiSengoku' (Kyushu 11), 'Kyushu 21', 'PI 417125', 'Kyushu 37', 'Kyushu 56', 'Tamahikari', 'Gakuran

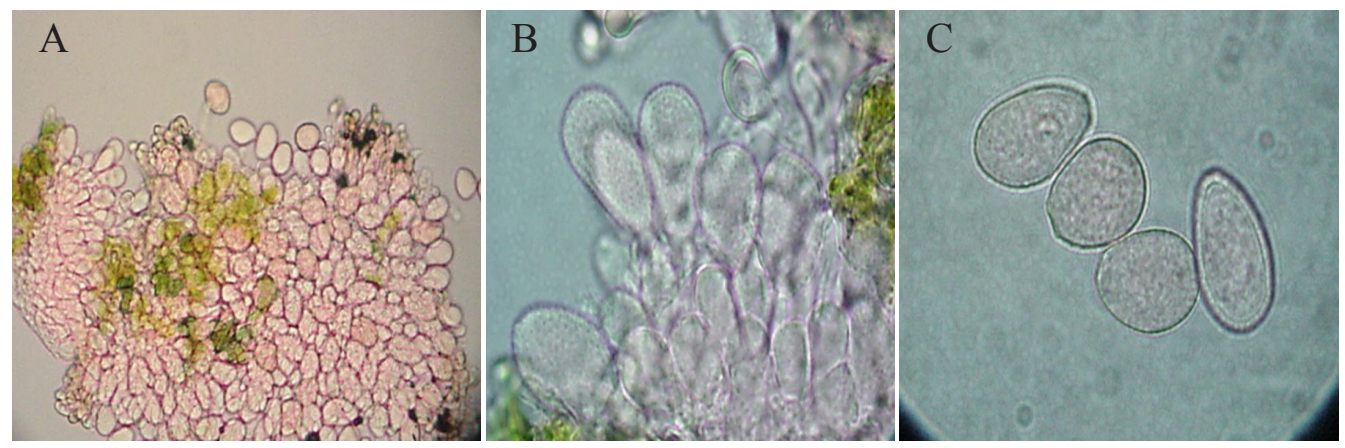

Figura 1. Pústulas $(\mathrm{A} ; 10 \mathrm{x})$, uredos $(\mathrm{B} ; 60 \mathrm{x})$ y urediosporas $(\mathrm{C} ; 60 \mathrm{x})$ de P. pachyrhizi, de hojas de soya infectadas y colectadas en el Estado de Nuevo León. 
Daizu,' 'Kumaji 2', 'Kuro Chouhin 14,' 'Kyushu 43', 'Ba yue bao,' 'PI 567024', 'PI 567025 A, 'PI 567123 A', 'Gogaku,' 'PI 605891 B', 'Kuro Daizu A, 'Kuro Daizu B', 'A9', 'PI 567058 D’ y 'PI 567104 B'. No se observó una reacción de severidad intermedia, ya que el resto de los genotipos evaluados (174) mostraron susceptibilidad. De esta respuesta podría inferirse que la virulencia de los aislamientos de $P$. pachyrhizi inoculados fue probablemente alta. Pham et al. (2009) demostraron que la reacción a roya en líneas de soya de Paraguay y Vietnam variaron considerablemente a la inoculación de 10 aislamientos de $P$. pachyrhizi procedentes de diversos países.

Es importante destacar que las líneas de soya 'PI 567123 A', 'PI 230970', 'PI 567058 D' y 'PI 567104 B', identificadas como resistentes en el presente estudio, también han sido registradas como resistentes con aislamientos de otras regiones del mundo. Esta característica puede conferir a las líneas mayor amplitud de resistencia a diferentes aislamientos de P. pachyrhizi. La línea 'PI 567123 A', también fue seleccionada resistente con la inoculación de colectas de P. pachyrhizi de Tailandia, Brasil, Paraguay y Zimbawe (Miles et al., 2006); 'PI 230970' mostró resistencia en Mississippi, EUA (Shuxian, 2009); Igualmente, 'PI 567058 D' y 'PI 567104 B' fueron reportadas como resistentes a 10 aislamientos de diferentes regiones donde la enfermedad es endémica (Pham et al., 2009). Esta es la primera información generada en México sobre la reacción de genotipos de soya a la roya asiática. Se concluye que se identificaron 23 genotipos de soya como resistentes a la roya asiática en condiciones de campo, los cuales podrían representar nuevas fuentes de resistencia a $P$. pachyrhizi y ser utilizadas como progenitores en el mejoramiento genético de la soya en México.

\section{AGRADECIMIENTOS}

Los autores agradecen la colaboración del Dr. Randall L. Nelson, curador de la colección de germoplasma de soya del Departamento de Agricultura de los Estados Unidos en Urbana, Ill., quien facilitó 132 genotipos para la realización del estudio. También agradecen al Fondo para el Fomento de la Producción de Semillas Oleaginosas en México de la industria del aceite y proteína (ANIAME, CAPRO y CANAJAD) por el financiamiento recibido para realizar la presente investigación.

\section{BIBLIOGRAFÍA}

Cárcamo-Rodríguez A. and J. Aguilar-Ríos (2006) First report of Asian soybean rust caused by Phakopsora pachyrhizi from Mexico. Plant Disease 90:1260 (abstract).

Bonde M. R., D. K. Berner, S. E. Nester and R. D. Frederick (2007) Effects of temperature on urediospore germination, germ tube growth, and initiation of infection in soybean by Phakopsora pachyrhizi. Phytopathology 97:997-1003.

Giesler L. J. (2009) Asian soybean rust. Disponible en: http://pdc.unl.edu/ agriculturecrops/soybean/soybeanrust/ (Junio 2012).

Kawuki R. S., P. Tukamunhabwa and E. Adipala (2004) Soybean rust severity, rate of rust development, and tolerance as influenced by maturity period and season. Crop Protection 23:447-455.

Koenning S., J. Mueller, R. Kemerait and P. Phipps (2008) Soybean Rust. Soybean Disease Information Note 8. North Carolina State University. Disponible en:www.ces.ncsu.edu/depts/pp/notes/ soybean/soy008/ (Junio 2012).

Maldonado-Moreno N. (1994) Huasteca 100 y Huasteca 200, nuevas variedades de soya para el sur de Tamaulipas. Folleto Técnico Núm. 9. Campo Experimental Sur de Tamaulipas, CIRNEINIFAP. Altamira, Tamaulipas, México.19 p.

Maldonado-Moreno N. y G. Ascencio-Luciano (2005a) Huasteca 300, nueva variedad de soya para el sur de Tamaulipas. Folleto Técnico Núm. 19. Campo Experimental Sur de Tamaulipas, CIRNE-INIFAP. Altamira, Tamaulipas, México.18 p.

Maldonado-Moreno N y G. Ascencio-Luciano (2005b) Huasteca 400, nueva variedad de soya para el sur de Tamaulipas. Folleto Técnico Núm. 18. Campo Experimental Sur de Tamaulipas, CIRNE-INIFAP. Altamira, Tamaulipas, México.18 p.

Miles M. R., R. D. Frederick and G. L. Hartman (2006) Evaluation of soybean germplasm for resistance to Phakopsora pachyrhizi. Plant Management Network 10:223-228.

Miles M. R., W. Morel, J. D. Ray, J. R. Smith, R. D. Frederick and G. L. Hartman (2008) Adult plant evaluation of soybean accessions for resistance to Phakopsora pachyrhizi in the field and greenhouse in Paraguay. Plant Disease 92:96-105.

Oloka H. K., P. Tukamuhabwa, T. Sengooba and S. Shanmugasundram (2008) Reaction of exotic soybean germplasm to Phakopsora pachyrhizi in Uganda. Plant Disease 92:1493-1496.

Pham T. A., M. R. Miles, R. D. Frederick, C. B. Hill and G. L. Hartman (2009) Differential responses of resistant soybean entries to isolates of Phakopsora pachyrhizi. Plant Disease 93:224-228.

Schneider R. W., C. A. Hollier, H. K. Whitam, M. E. Palm, J. M. McKemey, .R Hernández, L. Levy and P. R. DeVries (2005) First report of soybean rust caused by Phakopsora pachyrhizi in the continental United States. Plant Disease 89:774 (abstract).

Shuxian L. (2009) Reaction of soybean rust-resistant lines identified in Paraguay to Mississippi isolates of Phakopsora pachyrhizi. Crop Science 49:887-894.

SIAP, Servicio de Información Agroalimentaria y Pesquera (2014) Servicio de Información Agroalimentaria y Pesquera de la Secretaría de Agricultura, Ganadería, Desarrollo Rural, Pesca y Alimentación. Disponible en: www.siap.gob.mx/?option=com wrapper\&view=wrapper\&Itemid $=346$ (Enero 2014).

Terán-Vargas A. P., G. Ascencio-Luciano, N. Maldonado-Moreno and J. Ávila-Valdez (2007) La roya asiática de la soya en México. Folleto Técnico Núm. 22. Campo Experimental Sur de Tamaulipas, CIRNE-INIFAP. Altamira, Tamaulipas, México. 53 p.

Twizeyimana M., P. S. Ojiambo, T. Ikotum, C. Paul, G. L. Hartman and R. Bandyopadhyay (2007) Comparison of field, greenhouse, and detached-leaf evaluations of soybean germplasm for resistance to Phakopsora pachyrhizi. Plant Disease 91:1161-1169.

Yorinori J. T., W. M. Paiva, R. D. Frederick, L. M. Costamilan, P. F. Bertagnolli, G. E. Hartman and J. Núñez (2005) Epidemics of soybeans rust (Phakopsora pachyrhizi) in Brazil and Paraguay. Plant Disease 89:675-677. 